SUPPORTING INFORMATION

\title{
High density of aligned nanowire treated with polydopamine for efficient gene silencing by siRNA according to cell membrane perturbation
}

Baiju G Nair ${ }^{\mathrm{a}}$, Kyoji Hagiwara ${ }^{\mathrm{b}, \mathrm{c}}$, Motoki Ueda ${ }^{\mathrm{a}, \mathrm{b}}$, Hsiao-hua $\mathrm{Yu}^{\mathrm{a}, \mathrm{d}}$, Hsian-Rong Tseng ${ }^{\mathrm{e}}$ and Yoshihiro Ito $^{\mathrm{a}, \mathrm{b} *}$

${ }^{a}$ Nano Medical Engineering Laboratory, RIKEN, 2-1 Hirosawa, Wako, Saitama 3510198, Japan

${ }^{b}$ Emergent Bioengineering Material Research Team, RIKEN Centre for Emergent Matter Science, 2-1 Hirosawa, Wako, Saitama 3510198, Japan

${ }^{c}$ Laboratory of Human Science and Engineering, 1-3-1 Minaminagasaki, Toshima-ku, Tokyo 1710052, Japan

${ }^{d}$ Institute of Chemistry, Academia Sinica, 128 Academia Road Sec. 2, Nankang, Taipei 115, Taiwan

${ }^{e}$ Department of Molecular and Medical Pharmacology, University of California, Los Angeles CNSI, 570 Westwood Plaza, Los Angeles, CA 90095, USA

*Corresponding author.E-mail:y-ito@riken.jp 


\section{Quantification of siRNA conjugated on PD-SiNW substrates}

First, 1, 2, 3, 5 and $7 \mu \mathrm{g}$ FAM-siRNA in water was dropped onto various PD-SiNW substrates and dried in air without any disturbance. Subsequent fluorescent images of each PD-SiNW substrate were obtained by a molecular imaging system. Then, the intensity of fluorescence was calculated by ImageJ software. Finally, we plotted a standard curve in Microsoft Excel using the obtained values. Similarly, unknown siRNA concentrations on PD-SiNWs after washing were estimated from the standard curve using the image analysis and fluorescent intensity measurements. For example, the fluorescent intensity obtained from the siRNA-PD-SiNW surface was 5811 that corresponds to $2.79 \mu \mathrm{g}$ siRNA. Using this method, we also evaluated the amount of siRNA conjugated on PD-plain, plain, and SiNW substrates.

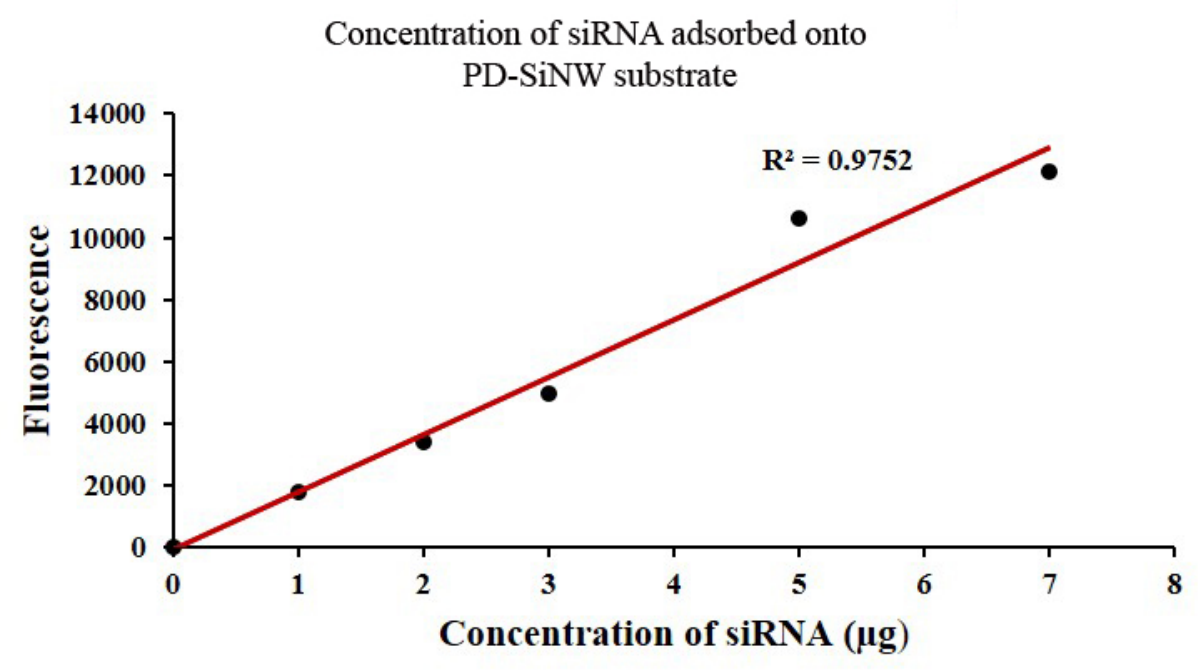

Figure S1 Standard plot obtained after image analysis of FAM-conjugated siRNA on PD-SiNW substrates. 


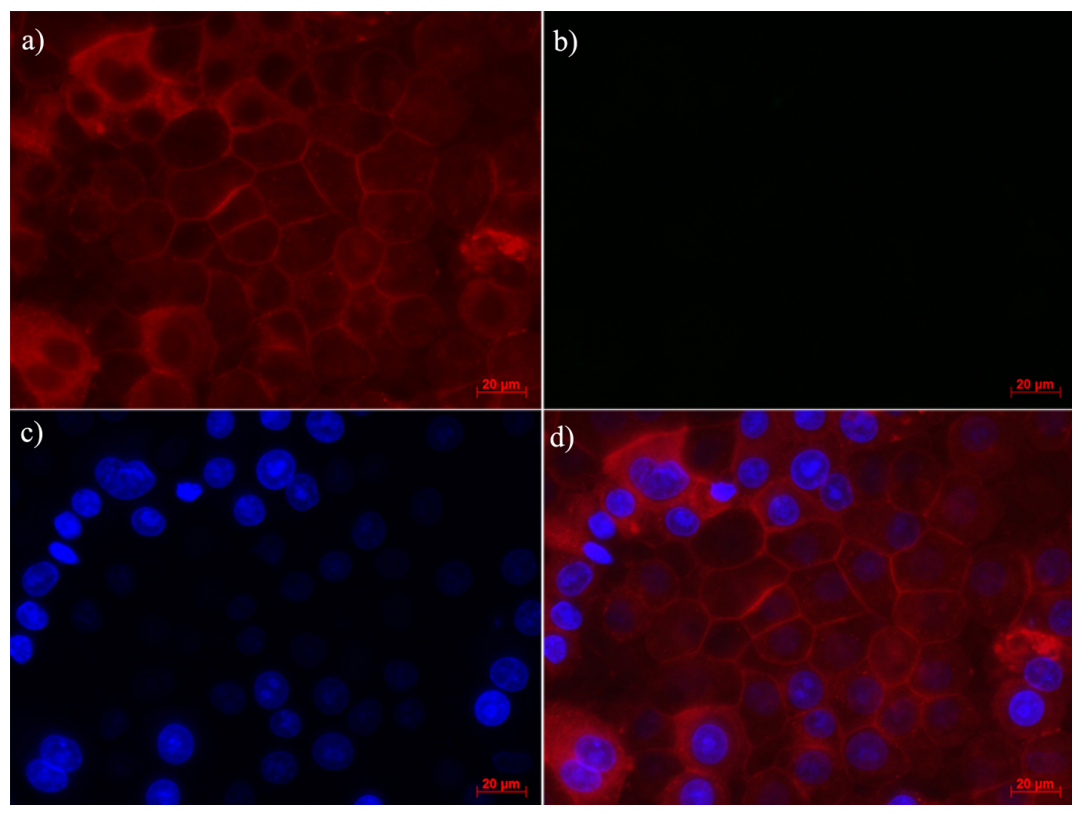

Figure S2 Fluorescent microscope images of A549 luc cells grown on a SiNW substrate. A549 luc cells were treated with (a) CellMask for plasma membrane staining, (b) FAM-siRNA, (c) Hoechst 33258 for nucleus staining, and (d) merged image. Scale bar is $20 \mu \mathrm{m}$.

\section{Adhesion}

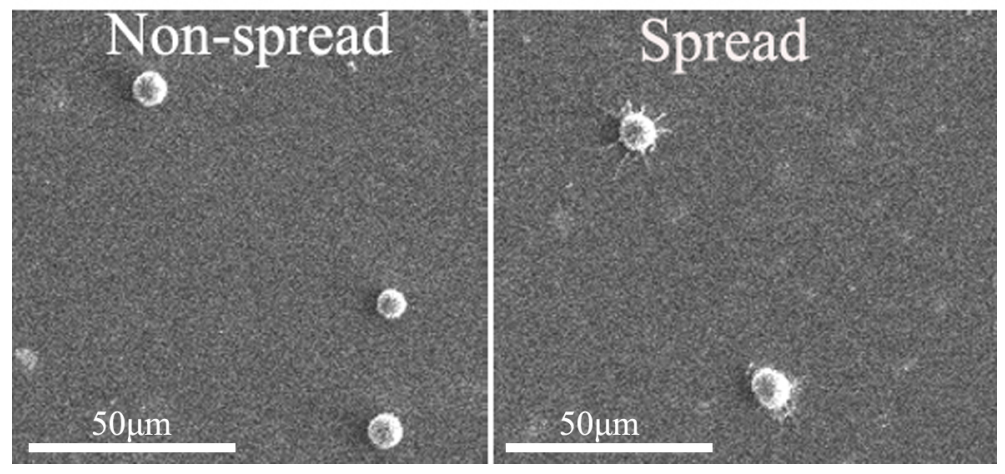

Figure S3 Spreading was determined by cell morphology. Cells with visible filopodia were counted as spread cells. 